\title{
La lucha por la ciudad: controversias en el proceso de formulación del Plan Cusco al 2025 - Movilidad y Espacio Público en una Ciudad Patrimonio (Cusco 2014-2018) (*)
}

\section{The fight for the city: controversies in the formulation process of the Cusco Plan to 2025 - Mobility and Public Space in a World Heritage City (Cusco 2014-2018)}

Claudia Alejandra Concha Vásquez $\left({ }^{* *}\right)$

Pontificia Universidad Católica del Perú

ORCID: 0000-0002-7706-290X

Fecha de recepción: 7 de febrero

Fecha de aceptación: 15 de junio

ISSN en línea: $2415-2498$

Concha Vásquez, C. (2018) «La lucha por la ciudad: controversias en el proceso de formulación del Plan Cusco al 2025 - Movilidad y Espacio Público en una Ciudad Patrimonio (Cusco 2014-2018)». Politai: Revista de Ciencia Política, Año 9, primer semestre, № 16: pp. 141- 176 .

DOI: https://doi.org/10.18800/politai.201801.005

* Una primera versión de este trabajo fue presentada en el $9^{\circ}$ Congreso de la Asociación Latinoamericana de Ciencia Política (ALACIP)

** Estudiante de último ciclo de Ciencia Política y Gobierno en la Pontificia Universidad Católica del Perú. Miembro del Grupo de Investigación en Instituciones, Políticas Públicas y Ciudadanía. Ha participado en el congreso de ALACIP del año 2017. Sus intereses de investigación son las políticas urbanas, culturas y desarrollo humano. claudia.concha@pucp.pe 


\section{Sumilla}

A través de la coordinación entre el Gobierno Regional de Cusco, la UNESCO y el Banco Mundial, nace el Plan de Movilidad y Espacio Público Cusco al 2025. Dicho plan tiene como objetivos ordenar el sistema vehicular de la ciudad, generar barrios donde la población pueda ocupar el espacio público disfrutando de las calles, parques y plazas, e implementar un nuevo modelo de transporte urbano compuesto por una ruta troncal con semaforización inteligente. El proceso de formulación del plan ha estado rodeado de conflictos técnicos, políticos y de coordinación entre las distintas instituciones implicadas. Además, entró en conflicto con los modelos de ciudad y los intereses de diferentes actores locales, tales como los vecinos del centro de la ciudad, las compañías de turismo, entre otros.

La siguiente investigación pretende identificar los factores que dificultaron el desarrollo del Plan Cusco al 2025, poniendo especial énfasis en las definiciones del problema público, las capacidades técnicas y políticas de las instituciones involucradas y la respuesta por parte de la prensa y la ciudadanía cusqueña. Ello nos permitirá ahondar en el estudio de la paradoja de aquellas iniciativas planificadas, que cuentan con apoyo político y económico por parte de instituciones locales e internacionales, pero aun así encuentran dificultades a lo largo de su desarrollo, lo cual tiene como consecuencia la implementación parcial o la cancelación de la política.

Palabras clave: Ciudades Patrimonio, políticas de movilidad, espacio público 


\section{Abstract}

Through coordination between the Regional Government of Cusco, UNESCO and the World Bank, the Plan for Mobility and Public Space Cusco 2025 was born. This plan aimed to order the vehicular system of the city, generate neighborhoods where the population can occupy the public space enjoying the streets, parks and squares, and implement a new urban transport model consisting of a main route with intelligent traffic lights. The process of formulating this plan has been surrounded by technical, political and coordination conflicts between the different institutions involved. Additionally, it came into conflict with the city models and the interests of different local actors, such as the residents of the city center, the tourism companies, among others.

This paper aims to identify the factors that hindered the development of the Cusco Plan by 2025, placing special emphasis on the definitions of the public problem, the technical and political capacities of the institutions involved as well as the response from the press and the citizens of Cusco. This, in turn, will allow us to delve into the study of the paradox of those initiatives which are planned and have political and economic support from local and international institutions, but still encounter difficulties throughout their development, which finally leads to partial implementation or the cancellation of the policy.

Keywords: World Heritage Cities, mobility policies, public space 


\section{Introducción}

La ciudad del Cusco fue declarada Patrimonio Cultural de la Humanidad el 9 de diciembre de 1983 por la Organización de las Naciones Unidas para la Educación, la Ciencia y la Cultura (Unesco). Esta designación significa que la ciudad de Cusco posee un gran valor por su historia, belleza y significado para la humanidad.

Los lugares considerados como Patrimonio Cultural de la Humanidad acceden a una serie de beneficios además del prestigio internacional. Tienen acceso a un fondo de 4 millones de dólares, el cual es destinado para asistir a los países en la identificación, promoción y preservación de estos sitios. En caso de desastres, estos sitios cuentan con la posibilidad de asistencia de emergencia para la reparación de daños. Adicionalmente, tienen acceso a la elaboración e implementación de planes para la administración del patrimonio, así como capacitación técnica brindada por expertos a los grupos encargados del cuidado de la ciudad dentro y fuera de las instituciones formales.

A pesar de ser una ciudad patrimonio y contar con todas las facilidades mencionadas anteriormente, la ciudad del Cusco presenta graves problemas a la hora de gestionar políticas de transporte. Uno de los problemas del sistema actual es la superposición de rutas en los principales corredores viales del centro. Esta superposición tiene como consecuencia que, dentro de la ciudad, y en especial en el casco histórico, confluyan varias rutas de diferentes empresas de transporte. Según el informe del Plan Cusco al 2025, se estima que el $90 \%$ de las rutas pasan necesariamente por el centro, lo cual genera una competición por el espacio público que se traduce en conflicto.

El perfil del sistema de transporte de la ciudad del Cusco, según el estudio presentado en el Plan Cusco al 2025, es un modelo urbano heredado y basado en una ciudad dispersa, lo cual se traduce en una falta de cobertura del transporte público, así como en la conformación precaria de una red vial externa que excluye a los peatones y usuarios de la bicicleta. Esta situación es el resultado de una disociación entre la visión del transporte, la gestión territorial y la escala que requiere la ciudad para el desplazamiento de las personas y el uso del espacio público. Asimismo, la infraestructura vial está 
incompleta. Encontramos tramos discontinuos, sin una lógica que se encuentre dentro de un planeamiento articulado de la ciudad. Esto, a su vez, se agrava por la poca inversión pública dentro de este rubro.

Dentro del análisis realizado en el Plan Cusco al 2025, se menciona que la caracterización global de la movilidad es singular en el Cusco. Casi el 50\% de la población se desplaza a pie, el 39\% en transporte público, el 3\% en transporte privado y el $10 \%$ en taxi. Sin embargo, casi todas las infraestructuras y recursos están destinados a ese 3\% de la población que se desplaza en transporte privado. Por lo tanto, es necesario dar un para que las políticas sean proporcionales a estas cifras y así el $50 \%$ de peatones reciban la prioridad en la inversión.

Otras de las características que hace especial a la ciudad del Cusco es su cercanía a sitios arqueológicos como Machu Picchu, Pisac, Moray, entre muchos otros. Esto ha posicionado al Cusco como una de las principales ciudades turísticas a nivel mundial. Considerando esta característica, se hace aún más evidente que la ciudad necesita de políticas de gestión del espacio y del patrimonio.

A un nivel más macro de análisis, el hecho de que la ciudad del Cusco sea declarada como Patrimonio de la Humanidad ha generado diferentes presiones sobre el gobierno local de la ciudad. Por un lado, este ha tenido que responder a un flujo no esperado de turistas que generan una demanda de servicios y necesidades que no se encontraban dentro del imaginario local. El turismo resulta ser una actividad que, si bien genera desarrollo económico, origina problemas de sostenibilidad ambiental, donde intereses privados son reacios a ceder espacio para la construcción de planes articulados sobre protección del patrimonio, movilidad y espacio público. Por otro lado, la presión sobre el gobierno local se ejerce también desde un ámbito internacional. Se espera que las competencias locales, a través del apoyo internacional, transformen el perfil de la ciudad del Cusco como una que sea capaz de responder las demandas de una ciudad cosmopolita, sin perder su identidad y características que la convierten en una ciudad única.

En respuesta a todo lo descrito anteriormente, organizaciones como el Banco Interamericano de Desarrollo y la Unesco realizaron el Plan Cusco al 2025-Movilidad y Espacio Público en conjunto con el Gobierno Regional y 
La lucha por la ciudad: controversias en el proceso de formulación del Plan Cusco al 2025 Movilidad y Espacio Público en una Ciudad Patrimonio (Cusco 2014-2018) - Claudia Concha

Vásquez

la Alcaldía de Cusco. Este plan nace como respuesta al crecimiento poblacional que demanda una mayor ocupación de suelo y a la necesidad de la integración del sistema de transporte público urbano con los subsistemas que dan servicio a las zonas rurales, asociado a una estratégica implementación de estacionamientos. Asimismo, responde a la necesidad de valoración del espacio público como regla general que conduce a un equilibrio óptimo en aspectos como la contaminación, el ruido y los accidentes que actualmente degradan la calidad de la vida urbana.

Los objetivos generales del Plan son construir una ciudad donde la gente se sienta a gusto, que pueda disfrutar de las áreas verdes, espacios deportivos y zonas culturales. Además, se busca desarrollar un perfil de ciudad incluyente, donde se potencien los transportes públicos que permitan la vida autónoma de todas las personas y accesibilidad universal. Se busca transformar la ciudad para convertirla en una habitable, donde los espacios públicos como las calles o las plazas no estén dominados por los vehículos.

El proceso de implementación del Plan Cusco al 2025 inició en mayo de 2015 con la restricción parcial del ingreso de unidades vehiculares a la Plaza Mayor de la Ciudad Imperial. A mediados de diciembre de 2016, se cerraron todos los ingresos a la plaza. Este era el segundo paso para conseguir que la zona monumental de la ciudad de Cusco sea de uso exclusivo para el peatón y, de paso, se proteja el patrimonio cultural que se ve afectado por el paso de los vehículos. Un año y siete meses después del proceso descrito anteriormente, el trabajo realizado hasta entonces se vio limitado por la decisión de algunos miembros del Consejo Municipal y un grupo de ciudadanos cusqueños. En la sesión de la tarde del martes 10 de enero del 2017, la mayoría de los consejeros municipales aprobó la derogación de la ordenanza $\mathrm{N}^{\circ} 011$ - 2015 que fomentaba el proceso de peatonalización del centro de la ciudad. El rechazo al proceso de implementación del plan no fue solamente de parte de los consejeros municipales. A través de un pronunciamiento público, la Cámara Regional de Turismo de Cusco (Cartur) expresó también su desacuerdo al denominado Plan Cusco 2025 sobre movilidad y espacios públicos.

A partir de lo presentado, se van construyendo una serie de cuestiones en las cuales podemos ahondar. ¿Cómo es que un plan realizado por especialistas, financiado por instituciones internacionales y apoyado por el Gobierno 
Regional presenta este nivel de rechazo al momento de su formulación? Esta investigación nos permitirá dilucidar y reconocer los vacíos dentro del diseño del Plan Cusco al 2025. Asimismo, reconoceremos una serie de actores con intereses que no coinciden necesariamente con las perspectivas presentadas en el plan y esto conlleva a que se dificulte la formulación. Este artículo representa un aporte al estudio y análisis de las Políticas Públicas, ya que permite comprender las deficiencias dentro de un plan que fue diseñado por especialistas y siguió los procesos recomendados a la hora de la construcción de una política de este tipo, pero aun así, en el momento de ponerlo en práctica, se encontró con distintos actores que rechazaron su implementación.

\section{Marco teórico}

Los lineamientos del Plan Cusco al 2025 - Movilidad y Espacio Público han sido diseñados bajo la perspectiva de organizaciones internacionales como el Banco Interamericano de Desarrollo (BID) y la Organización de las Naciones Unidas para la Educación, la Ciencia y la Cultura (Unesco). Por lo tanto, conocer la definición que manejan estas organizaciones sobre la ciudad sostenible es importante para comprender las visiones contenidas dentro del plan.

Para el BID, una ciudad sostenible se define como aquella urbe que ofrece una alta calidad de vida a sus habitantes y que minimiza sus impactos en el medio ambiente. Asimismo, es aquella ciudad que cuenta con un gobierno local con capacidades físicas y administrativas para mantener su crecimiento económico y para llevar a cabo sus funciones urbanas con la participación ciudadana, con el objetivo de beneficiar a futuras generaciones. La ciudad sostenible debe sobresalir en tres dimensiones: una dimensión de sostenibilidad ambiental y de cambio climático, una segunda dimensión de desarrollo urbano sostenible y una tercera dimensión que abarca la sostenibilidad fiscal y gobernabilidad.

La dimensión ambiental incluye temas como la calidad del aire y el agua, la mitigación de emisiones de gases de efecto invernadero, la adaptación al cambio climático, la reducción de la vulnerabilidad ante los desastres 
naturales y la cobertura de los servicios públicos. La dimensión del desarrollo urbano considera los aspectos físicos, económicos y sociales del desarrollo. Sobre esta dimensión, la Unesco menciona que la cultura es un recurso clave para promover el desarrollo urbano sostenible, para el mantenimiento de la identidad urbana y el medio ambiente. Finalmente, la tercera dimensión aborda las características de la buena gobernabilidad, tales como la transparencia, la participación pública y la gestión orientada a la obtención de resultados, así como también las buenas prácticas fiscales de las ciudades.

Para la UNESCO, los enfoques que se deberían considerar para construir ciudades sostenibles son la integración de las políticas urbanas a nivel nacional, las reformas institucionales y legales, y la ampliación de alianzas con todos los actores relacionados al tema. La conservación y protección del patrimonio material e inmaterial son el punto de partida para una estrategia para el desarrollo urbano sostenible. Para la Unesco, la conservación del patrimonio es trascendental para la construcción de ciudades sostenibles, no solo por un criterio valorativo, sino también económico. La vitalidad cultural de una ciudad le permite diferenciarse de las demás, posicionarse en el ámbito nacional o internacional y mejorar así su atractivo para los inversores. Las industrias culturales y el turismo sostenible basado la revitalización urbana están entre los sectores económicos más poderosos para crear empleos verdes, estimular el desarrollo local y fomentar la creatividad.

A partir de lo mencionado anteriormente, desarrollamos el estado de la cuestión de la presente investigación siguiendo las cuatro líneas conceptuales desarrolladas a continuación:

\section{a) Ciudades intermedias, patrimonio y turismo sostenible}

Consideraremos a la ciudad del Cusco como una ciudad intermedia. Dichas ciudades, según el trabajo de Bueno, se caracterizan por su interacción constante y duradera con su espacio regional, así como con las aglomeraciones urbanas de jerarquía superior (Bueno, citado por Stadel 2014). Mantienen un tamaño demográfico funcional, suficiente para poder ofrecer bienes y servicios al espacio microregional al cual están ligadas. Asimismo, tienen capacidad para recibir migrantes de ciudades menores, lo cual permite establecer relaciones 
dinámicas con el espacio rural microrregional que las envuelve. Presentan una clara diferenciación del espacio intra-urbano con un centro funcional individualizado y una periferia dinámica envolvente.

Debido a estas características, la Ciudad del Cusco, así como la mayoría de las ciudades intermedias, «ofrecen más ventajas para la localización de negocios que las capitales nacionales debido a su proximidad a los recursos, a los corredores nacionales o a grandes aglomeraciones metropolitanas» (Stadel 2014). En el caso de Cusco, especialmente, su proximidad a sitios arqueológicos ofrece ventajas para el desarrollo de actividades relacionadas al turismo. El dinamismo de dicha actividad configura el perfil del espacio físico donde se desarrolla. Debido a esta interacción, «algunas ciudades intermedias están sufriendo de una deficiencia muy marcada en el área de la administración urbana, lo que significa que los niveles de productividad, capital y sustentabilidad medio ambiental, parecen aún menos favorables que en el caso general de los grandes centros urbanos». (Stadel 2014)

El perfil de un ciudad intermedia tiene una escala apropiada para la participación en los asuntos comunitarios, mejores condiciones físicas en cuanto a su calidad de vida, una más adecuada escala humana para los recorridos diarios y distancias centro-periferia, mejor equilibrio entre el presupuesto y el tiempo de viaje cotidianos, una más fácil accesibilidad al campo como lugar de recreación, menor contaminación del aire y menor nivel de ruido, y un mayor control social que se traduzca en mayor índice de seguridad personal (Bueno, citado por Stadel 2014).

Esta situación se encuentra intrínsecamente relacionada con la designación de la ciudad del Cusco como una ciudad patrimonio. Ser una Ciudad Patrimonio significa que dicha ciudad posee un gran valor por su historia, belleza y significado para la humanidad. Asimismo, implica una serie de beneficios, tanto económicos como de capacitación técnica en temas de protección y gestión del patrimonio, por parte de la Unesco. Sin embargo, el problema de dicha designación es que puede resultar contraproducente para la ciudad, ya que cada vez está siendo más notorio que las ciudades que cuentan 
con un rico patrimonio arquitectónico y monumental inicien una especie de «matrimonio de conveniencia» con el turismo, tal como nos menciona Javier Melgosa. Este «matrimonio» puede generar «impactos negativos en el patrimonio histórico y en el ambiente urbano» (Melgosa 2001). Por ello, resulta necesario buscar un equilibrio entre desarrollo turístico y conservación, ya que puede llegar el caso de que algunas ciudades no puedan soportar el creciente número de visitantes.

Los impactos sobre las condiciones naturales son aquellos «producidos sobre las condiciones naturales, la trama urbana, patrimonio urbanístico y arquitectónico, y sobre el entorno» (Troitiño, 2011). Con efectos sobre la trama urbana, la autora se refiere a la «invasión de calles y plazas por vehículos circulando o aparcados que suele ser un foco de conflictos» (Troitiño 2011). Así, «cuando las calles y plazas coinciden con las rutas turísticas y los ámbitos más visitados, el centro histórico turístico puede llegar a convertirse en una prolongación del espacio comercial a través de estantes, mostradores, escaparates y exhibidores de los artículos que se ofrecen o verse ocupados por kioscos de venta, terrazas y veladores de los establecimientos de restauración» (Troitiño 2011). Con impactos sobre el patrimonio urbanístico y arquitectónico, la autora se refiere a obras de nueva planta dedicadas a satisfacer la demanda turística, tales como hoteles, restaurantes, edificios comerciales, entre otros.

Luego de mostrarnos los principales impactos que pueden afectar a las Ciudades Patrimonio, la autora nos presenta algunas recomendaciones para desarrollar un turismo sostenible dentro de estas ciudades en tres ejes: la esfera física y urbanística, la esfera medioambiental y la movilidad.

Dentro de la política general referida a la esfera física y urbanística, recomienda «hacer un mejor uso de la estructura física, rediseñando nuevas formas de uso del espacio colectivo, reduciendo el espacio ocupado por automóviles o por los usos turísticos allí donde resulten agresivos», así como «el refuerzo del transporte colectivo e incremento de zonas peatonales» (Troitiño, 2011). 
Respecto a la esfera medioambiental, recomienda «desarrollar políticas para proteger la calidad del espacio urbano y de sus entornos, evitando la contaminación visual o paisajística. Desarrollar medidas para evitar la contaminación acústica y fijar los niveles máximos de ruido. Estudiar la posibilidad de establecer una "ecoetiqueta" a aquellas ciudades históricas que mejor resuelvan las relaciones entre el turismo, el patrimonio cultural, el medio ambiente y la calidad de vida». (Troitiño 2011)

Las recomendaciones en relación con la movilidad son «desarrollar y aplicar planes de movilidad orientados a reducir la movilidad motorizada de los centros históricos, manteniendo al mismo tiempo adecuadas condiciones de accesibilidad» (Troitiño 2011). La autora recomienda también «medidas de peatonalización o de calles de coexistencia, pero siempre dando prioridad al peatón sobre el automóvil, en los principales itinerarios turísticos y comerciales de los cascos históricos», «prohibición del aparcamiento en el entorno de los edificios monumentales y a lo largo de los principales itinerarios peatonales» y «reforzar y mejorar los sistemas de señalización a la entrada de las ciudades históricas para canalizar los vehículos de los visitantes hacia los aparcamientos existentes o hacia zonas donde no generen impactos negativos» (Troitiño 2011).

Siguiendo estas recomendaciones, el turismo, bien organizado, no solo tiene un papel destacado en el desarrollo económico local, sino que también contribuye a la valoración, conservación y adecuación para la visita turística de parte del patrimonio cultural.

\section{b) Ciudades sostenibles}

Jan Bazants define una ciudad sostenible como una urbe que tiene la capacidad para atender y satisfacer las necesidades de sus ciudadanos, considerando el devenir de las generaciones futuras (Bazants 2009). Este autor menciona, además, que la sostenibilidad consta de tres dimensiones: social, económica y ambiental. De la misma manera, Stadel señala que el desarrollo sustentable es la integración de los aspectos económicos, sociales y ambientales a fin de producir bienes 
y servicios, pero a la vez, preservar la diversidad y respetar la integridad funcional de los ecosistemas (Stadel 2014). Además, el autor agrega que la sustentabilidad requiere de una sociedad informada y con preferencias ambientales claras.

Dentro de las nuevas prácticas del desarrollo integral de la comunidad, autores como Stadel incluyen criterios como el valor de los individuos, la familia y la comunidad como miembros responsables y participantes de la sociedad. El propósito de estas prácticas es fomentar comunidades estables e independientes caracterizadas por una sensibilidad para las necesidades sociales y económicas en su orden de prioridades. Además, se enfoca en la importancia de la participación local y la movilización de los recursos materiales y humanos locales en todos los niveles del proceso de desarrollo (Stadel 2014).

Respecto a la sustentabilidad en el ámbito urbano, el autor involucra una dimensión retrospectiva, en un esfuerzo por mantener y restaurar los valores tradicionales de la ecología urbana y proteger o revitalizar el patrimonio urbano. Estos valores tradicionales son elementos esenciales para fortalecer la identidad local y el respeto en la población (Stadel 2014).

En el concepto de la sustentabilidad urbana, también se involucra una dimensión espacial a varios niveles, desde el nivel local de las comunidades y los barrios, hasta los niveles internacionales de las redes urbanas de comunicación (Stadel 2014). Respecto a esta dimensión espacial, existen criterios para evaluar la sustentabilidad en los barrios, los cuales son la organización base de toda ciudad. Juan Blanco Moya, en un estudio acerca de los barrios sustentables en Chile, se basa en los autores Kellett y Haapio para presentarnos los siguientes criterios que debería abarcar cualquier sistema de evaluación para barrios, independiente del contexto geográfico, político y social: un terreno sustentable, el uso de suelo previo y la reducción de expansión urbana, un sistema de transporte diseñado para incorporar al peatón, a los ciclistas y al transporte público, gestión de residuos y eficiencia en la producción de energía, y prioridad por preservar la biodiversidad (Blanco 2015). Asimismo, el 
autor incluye dentro de estos criterios de evaluación otros factores vinculados a la población, como por ejemplo la sustentabilidad económica de negocios, el empleo, las nuevas oportunidades y el bienestar entendido como calidad de vida (Blanco 2015).

En el caso de la ciudad del Cusco, así como en diferentes ejemplos de ciudades con rezagos rurales, los procesos de consolidación urbana se han dado de espaldas a la ruralidad. Esta situación ha contribuido al déficit ambiental y a la deuda ecológica local y regional. Según lo mencionado en el libro Carta Medellín: sobre el porvenir urbano de las urbes del mundo, lograr una ciudad sostenible implica identificar y gestionar la interrelación urbano-rural (Instituto Social de Vivienda y Hábitat Alcaldía de Medellín 2014). Para una articulada planificación urbano-rural en gestión territorial, es fundamental que se realicen y cualifiquen los estudios técnicos y científicos sobre este aspecto.

A modo de respuesta, la cumbre de las ciudades de las Naciones Unidas de 1996 ha sugerido normas básicas para la construcción del desarrollo urbano sostenible, además de medidas legislativas, de regulación y planificación y de inversiones públicas. Es necesario tomar en cuenta los buenos ejemplos que podemos identificar alrededor del mundo en relación a la gestión de las ciudades sostenibles. En ese sentido, Girardet señala que «compartir las mejores prácticas entre ciudades es un instrumento esencial para el desarrollo sostenible; aprendiendo del ejemplo la transformación local puede conducir al cambio global» (Girardet 2001). Sin embargo, el autor señala también que es necesario adaptar las políticas al contexto local. «Para que las buenas prácticas urbanas sean transferibles de una ciudad a otra, la acción debe ajustarse especialmente a las acciones locales» (Girardet 2001)

Luego de haber revisado literatura sobre la definición y aspectos a considerar para la construcción de una ciudad sostenible, pasaremos a presentar indicadores de sostenibilidad urbana presentados en el libro Estrategias medioambientales y sostenibilidad urbana (Casanueva y Fernández 2010). El conjunto de indicadores se estructuran en siete grandes apartados: la morfología urbana, el espacio público, la movilidad y los servicios, la complejidad urbana, el metabolismo 
urbano, el aumento de la biodiversidad y la cohesión social. El diseño y planificación de las ciudades deberán ser influenciados por cada uno de los indicadores presentados.

Una movilidad sostenible exige un nuevo modelo de movilidad y espacio público que organice la funcionalidad de viario público, incrementando las oportunidades de los medios de transporte alternativos y recuperando los espacios públicos como lugar de encuentro y espacio de convivencia. En ese sentido, «los nuevos procesos urbanísticos deberían reservar como mínimo el $30 \%$ de espacio libre al interior de una manzana», ya que «estos espacios garantizan la continuidad del espacio calle y la calidad del espacio abierto y permite múltiples usos comunitarios» (Casanueva y Fernández 2010).

En relación a los indicadores de movilidad y servicios, los medios de transporte público tienen un papel clave en el modelo de movilidad sostenible. «Se considera que existe una buena accesibilidad cuando desde cualquier parte del viario público puede accederse en 5 minutos a una parada de transporte público; se considera de acceso aceptable aquel que permita que toda la ciudadanía pueda acceder a una red de bicicletas en menos de un minuto en bicicleta o de 5 minutos a pie» (Casanueva y Fernández 2010).

Uno de los primeros casos de la construcción de una ciudad sostenible fue la ciudad de Curitiba (Brasil) en 1971. Una de las principales estrategias desarrolladas por el gobierno local de dicha ciudad fue la restauración del sistema de transporte público. «Esta medida, además de disminuir el uso del automóvil, trajo consigo la reducción de contaminación atmosférica y auditiva. Se estima que en 1991, cerca del $28 \%$ de los ciudadanos, motivados por la eficiencia del transporte público dejaron de usar su vehículo de manera regular» (Martínez 2012).

\section{c) Políticas de movilidad urbana y espacio público}

Las políticas de movilidad urbana y espacio público no estuvieron siempre en la agenda pública como prioridad. No fue sino hasta 
inicios de los años 80 que en América Latina el Estado acogió el tema dentro de la agenda política. A partir de la década de los 80, en el contexto de redemocratización de América Latina, se observa la búsqueda de una mayor representación política a través de una aproximación entre política y ciudad. Esto se confirma en el fortalecimiento del poder local, en el nuevo rol de la ciudad en el mundo, en el desarrollo de procesos de descentralización y en la apertura de nuevos canales de participación. (Carrión 2007). Asimismo, el desarrollo de procesos de descentralización dio paso al fortalecimiento de los poderes locales a través del surgimiento de nuevos actores locales y el aumento de recursos económicos.

Sin embargo, en nuestro país, esta nueva hegemonía de lo municipal y de los poderes locales no aseguró que se privilegiaran los temas de movilidad sostenible. Estos temas adquirieron relevancia a través de la discusión acerca del transporte.

Dextre menciona que el crecimiento del parque automotor sin planificación genera algunas externalidades como contaminación, congestión y accidentes que contribuyen al deterioro de la calidad de vida. Estos problemas originan «una presión pública que por lo general está orientada a la construcción de pasos a desnivel, nuevas vías o la ampliación de carriles y nuevas zonas de estacionamientos; medidas que tienen la finalidad de acomodar a los nuevos vehículos que ingresan al sistema vial» (Dextre 2001). Ante esto, el autor aconseja a los gobiernos locales que deben definir sus políticas de transporte pensando en una estrategia de desarrollo sostenible; es decir, cuando se apuesta en invertir en políticas de transporte sostenibles y planificadas obtenemos una tasa más alta de calidad de vida en las ciudades, se promueve el desarrollo económico de estas y se logra que las economías rurales sean saludables. En este sentido, reducir el impacto medioambiental del transporte y reducir la necesidad que tienen las personas de viajar en automóviles particulares se convierten en los pasos trascendentales para iniciar la construcción de ciudades sostenibles.

La reducción del impacto medioambiental del transporte se puede lograr «promoviendo medios alternativos de viaje, para lo cual será 
necesario trabajar en tres áreas: mejorar el conocimiento y responsabilidad de los usuarios, mejorar el transporte público y diseñar facilidades para los peatones y ciclistas»(Dextre 2001). El desarrollo de un sistema de transporte sostenible está basado principalmente en mejorar el conocimiento y responsabilidad de los usuarios y promover el uso del transporte público. Las características que debe tener un buen servicio de transporte público se puede lograr en la medida que se mejore la Gestión de las Empresas de Transporte Público, es decir, «es necesario que los operadores del transporte público se agrupen en asociaciones aportando sus vehículos a la empresa, de tal manera que todos tengan los mismos objetivos» (Dextre 2001). Por otro lado, el autor menciona que la mejor forma de formalizar a las empresas de transporte es mediante la licitación de las rutas de transporte público por parte de los gobiernos locales, teniendo como requisito que las empresas que se presenten a la licitación sean sociedades. Por otro lado, para que los usuarios elijan utilizar el transporte público por encima del privado, se debe invertir en la infraestructura vial; de esta manera se garantizará que los viajes sean en tiempos programados. Otra de las estrategias que presenta el autor es impulsar los transportes alternativos y construir una ciudad amigable para el peatón. Esto se logra a partir de diseñar infraestructura considerando las necesidades según la demanda de cada usuario.

Las políticas convencionales de gestión del tráfico han intervenido a través del modelo de la demanda solvente, por la cual se han construido todas las nuevas infraestructuras necesarias para cubrir la creciente demanda de transporte motorizado privado (vías rápidas circunvalaciones). Estas acciones, sin embargo, estimulan el proceso de motorización y se muestran insuficientes para dar respuesta real al problema, puesto que a medio plazo se retorna a la situación de congestión inicial. Revisando bibliografía sobre políticas de transporte en Perú se puede concluir que estas se diseñan pensando únicamente en los vehículos motorizados y como resultado de esto se aprecia que cada día es más difícil tanto para los peatones como para los ciclistas movilizarse dentro de la ciudad.

Córdova Aguilar, sobre el transporte urbano en Lima, menciona que 
en «las ciudades se concentran tres tipos fundamentales de transporte: el de pasajeros, mercancía y el privado. Cuando analizamos el transporte urbano debemos tener en cuenta los tipos de vehículos, la infraestructura y las operaciones que se requieren» (Córdova 2013). Actualmente, las ciudades deben atender las demandas de redes de transporte capaces de movilizar gran cantidad de población. Esto lleva a la búsqueda de estrategias que mejoren la eficiencia y capacidad de atención a la población. El autor identifica tres principales frenos para la fluidez del transporte: un sistema de transporte público inadecuado, la gran concentración del tráfico en las principales avenidas y la contaminación ambiental por la concentración vehicular (Córdova 2013). Para mitigar la congestión del tránsito, recomienda un mejor control de las señales de tránsito, mejoramiento de intersecciones, administración de demanda de tránsito, seguridad vial e inspección vehicular. (Córdova 2013).

\section{d) Globalización y ciudades}

La coyuntura urbana de América Latina ha ido cambiando en relación a grandes acontecimientos urbanos. Las perspectivas que han dominado a las ciudades no son ajenas a los procesos de democratización y a la globalización. Dicha coyuntura ha pasado de la etapa iniciada en la segunda posguerra, con una lógica de urbanización basada en la periferización popular (favelas, villas, pueblos jóvenes) y la metropolización industrial propia del modelo de sustitución de importaciones y del Estado de Bienestar, hacia otra etapa basada en un nuevo patrón de urbanización sustentado en el regreso a la ciudad construida y la ciudad en red, en un contexto de globalización (Carrión 2007).

Debido al paso de la globalización sobre las ciudades, se ha formulado una nueva forma de entender la ciudad y los poderes locales. Los principales hechos que apoyan dicho cambio de paradigma son la transición demográfica que urbaniza el continente, la globalización que impulsa la internacionalización (privatización, ajuste y apertura) y los procesos políticos (descentralización, representación e instituciones) (Carrión 2007). 
Al mismo tiempo que los procesos mencionados anteriormente, el perfil de América Latina a partir de los años 80 cambió drásticamente. Un ejemplo de ello es que el ciclo de migración cambió; pasó a ser de una urbe a otra, mientras que antes se daba la migración del campo a la ciudad. Esto generó que el foco de atención regrese a las ciudades, pues se presentaron nuevas demandas sociales urbanas.

Los modelos políticos para gobernar las ciudades también se vieron influenciados por los procesos de globalización, democratización y neo-liberalización que se dieron en América Latina durante las décadas de los 80 y 90 . Se perciben dos modelos políticos de gobierno de la ciudad: el de la ciudad empresarial privada, que recurre al mercado para inyectarle eficiencia a la gestión urbana, y el de la ciudad inclusiva, basada en una revalorización del espacio público y la promoción de derechos. (Carrión 2007).

Respecto a este tema, Parnreiter reconoce un tercer modelo político y social urbano: la ciudad global (Parnreiter 2002). Este modelo se refiere a una forma de centralidad urbana donde se integran economías regionales, nacionales e internacionales. Dentro de las ciudades globales, la variable decisiva son las economías globales, pues son las que establecen un proceso de creciente interdependencia entre las ciudades, un proceso que debe ser entendido en el contexto de una red global urbana (Chion 2002). Al formarse la red global urbana, Chion propone un análisis del espacio urbano metropolitano que deja de tener el foco solamente en un lugar priorizar la interacción entre lugares (Chion 2002).

En el caso peruano, a partir de los años 90, el contexto sociopolítico sufrió grandes cambios. Debido a políticas neoliberales y a la implementación de políticas económicas que favorecían inversiones internacionales, las ciudades en Perú rompieron su aislamiento de la economía global y retomaron su participación en las redes globales. Estos cambios políticos y económicos tuvieron una incidencia concreta en la reorganización de actividades urbanas y su configuración espacial (Chion 2002).

La movilidad espacial de la población cambió. Antes era mayormente 
concéntrica, de lugares residenciales a determinados centros de empleo y de servicios ubicados alrededor del Centro Histórico. A partir de los 90, la movilidad se realiza dentro de un patrón multidireccional entre los múltiples centros especializados (Chion 2002). Estos centros de los que habla el autor han generado posibilidades de desarrollo metropolitano, donde la habilidad de los actores locales para potenciar los procesos de integración espacial será esencial para incrementar las ganancias económicas. Además, el desarrollo de centros especializados puede facilitar la convergencia de múltiples grupos sociales. Esta convergencia, a su vez, genera espacios de identidad cultural, dinámica que es atractiva para el visitante internacional y el desarrollo del turismo.

Por otro lado, Mattos señala que, al contrario de lo que se creería, la globalización no implica que desaparezca el conjunto de rasgos inherentes a la identidad básica de cada ciudad. Por el contrario, en virtud de este proceso, cada ciudad se transforma preservando muchos de los rasgos establecidos a lo largo de su historia, que son los que la distinguen de otras ciudades de su mismo ámbito geográfico. Estos rasgos están relacionados con la identidad histórica de cada ciudad (en la que sin duda tienen gran importancia la idiosincrasia de sus habitantes, la morfología original del lugar de emplazamiento, las modalidades de la vida urbana cotidiana que fueron siendo establecidas), la cual seguramente permanecerá más allá de los impactos de la globalización (Mattos 2002). Además de los cambios culturales, Veltz nos menciona los cambios en la organización y el funcionamiento de la ciudad, los cuales estarían apareciendo en las ciudades más tocadas por la globalización y que se derivarían de la formación y consolidación de una nueva base económica metropolitana altamente tercerizada, así como de la consecuente estructuración de una nueva arquitectura productiva (Veltz 2000). 


\section{Metodología}

En esta investigación se ha empleado una metodología cualitativa que contempla el proceso de formulación del Plan Cusco al 2025 - Movilidad y Espacio Público desde año 2015 hasta el 2018. Se ha elegido ese rango de años debido a que los principales hitos del diseño de la política corresponden a este tiempo (ver línea de tiempo al final de esta sección).

Durante el trabajo de campo, se realizó entrevistas semiestructuradas a los actores relevantes en la formulación del plan. Para efectos del análisis de actores, encontramos pertinente apoyarnos en Dente y Subirats. Los actores, según estos autores, son aquellos que llevan a cabo las acciones relevantes (Dente y Subirats 2014). Los actores no son necesariamente individuos, sino que también pueden ser organizaciones, siempre y cuando «haya una coherencia interna suficiente y un control colectivo de los recursos utilizados» (Dente y Subirats 2014). Dichos actores tienen, además, objetivos e intereses. Para ello, necesitan recursos. Sobre este tema, Dente y Subirats señalan que existen cuatro tipos de recursos: políticos, económicos, legales y cognitivos (Dente y Subirats 2014).

Los recursos políticos consisten en la cantidad de consenso que un actor es capaz de poner en movimiento. El nivel de consenso del que goza un actor o un determinado planteamiento puede comprobarse mediante las elecciones o los referéndums, puede ser modificado a través de campañas de información y comunicación y suele verse afectado por acontecimientos externos (Dente y Subirats 2014). Los recursos económicos incluyen la capacidad de movilizar recursos económicos de cualquier tipo para modificar o influenciar los comportamientos de otros actores (Dente y Subirats 2014). Los recursos legales: consisten en las potestades o posiciones de ventaja que las normas jurídicas y administrativas atribuyen a algunos sujetos (Dente y Subirats 2014). Finalmente, los recursos cognitivos consisten en la disponibilidad de información o de modelos interpretativos relevantes para la adopción de una decisión (Dente y Subirats 2014).

En base a ello, se seleccionó los siguientes grupos de actores para esta 
investigación: funcionarios (trabajadores de la Municipalidad del Cusco y del Gobierno Regional), políticos (regidores de la municipalidad), expertos (coordinador del equipo de trabajo del plan, consultores del Banco Mundial) y sociedad civil (prensa, vecinos y empresarios del Centro Histórico, Cámara de Comercio del Cusco y Cámara de Turismo del Cusco).

De manera complementaria a las entrevistas, se realizó un análisis de la prensa local. Para ello, se tomó en cuenta los tres periódicos más leídos en el Cusco según el estudio realizado por la Compañía Peruana de Estudios de Mercados y Opinión Pública 2016. Estos periódicos son El Sol del Cusco, El Diario y El Comercio (edición para Cusco).

Para orientar el proceso de revisión de prensa, se ha realizado una línea de tiempo con las fechas claves dentro del proceso de formulación del plan. Dichas fechas se usarán como referencia para buscar noticias relacionadas quince días antes y quince días después del suceso. De esta manera, se podrá apreciar mejor el escalamiento de los conflictos, así como una mejor descripción de los actores y sus perspectivas del problema público. Tal como se puede observar en la línea del tiempo que se presenta a continuación, las fechas seleccionadas son los meses de octubre, noviembre y diciembre del año 2016, enero, febrero, marzo, abril, mayo y diciembre del año 2017, y enero y febrero de 2018. Se utilizarán las noticias descriptivas y no los artículos de opinión, pues se desea conocer lo que la prensa presenta como la verdad. Lo que se busca identificar dentro de las noticias son los actores más relevantes en el suceso representado y los principales argumentos en relación a la formulación del plan. 
La lucha por la ciudad: controversias en el proceso de formulación del Plan Cusco al 2025 Movilidad y Espacio Público en una Ciudad Patrimonio (Cusco 2014-2018) - Claudia Concha
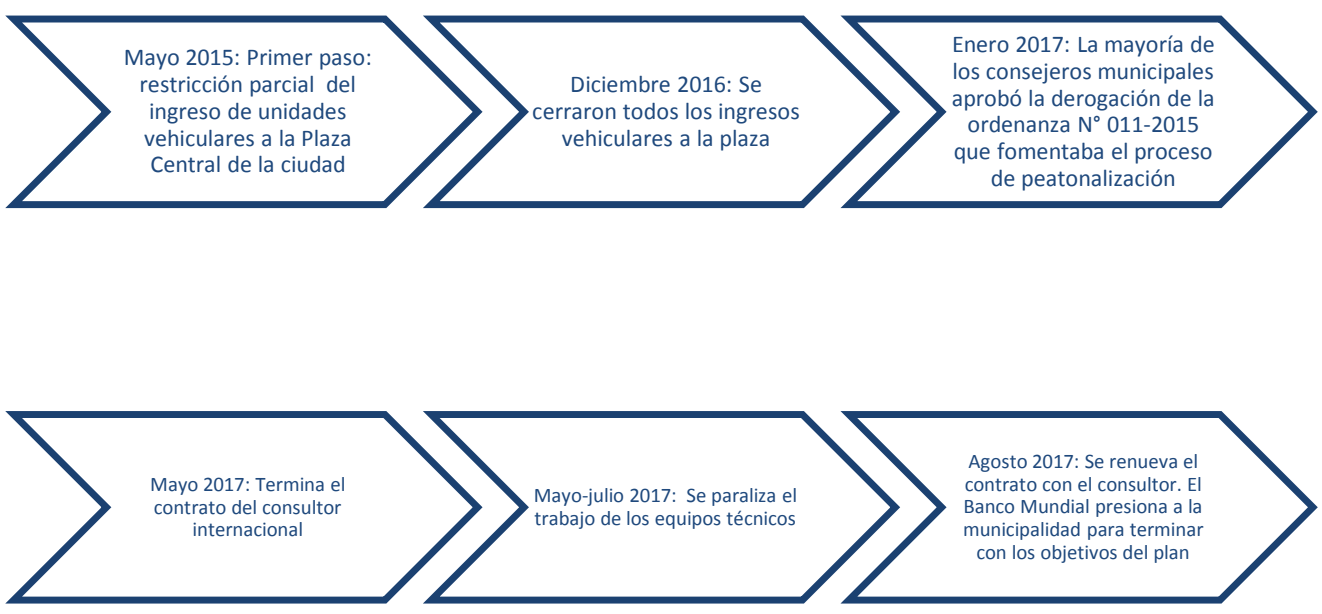

Gráfico 1. Línea de tiempo del proceso de formulación del Plan Cusco al 2025 Movilidad y Espacio Público.

\section{Descripción del caso}

El primero de enero del año 2015, el abogado Carlos Moscoso Perea juramentó como alcalde de la ciudad del Cusco. En su primer discurso como burgomaestre, sostuvo que en los cien primeros días de gestión crearía el Instituto de Planificación y Urbanismo, así como un centro cultural y el fondo de salvamento de Cusco para proteger el patrimonio. Es así que, desde el inicio de su gestión, mostró un especial interés por los temas de planificación urbana y gestión de los espacios públicos. Como muestra de ello, se pueden encontrar siete planes de ordenamiento urbano: el Plan de Desarrollo Metropolitano de la ciudad de Cusco, el Plan de Desarrollo Urbano, el Plan de Desarrollo Distrital (encargado de temas más puntuales como estacionamiento público, ornato y mobiliario urbano), el Plan Maestro del Centro Histórico (plan orientado a la efectiva protección y revitalización del Centro Histórico de Cusco), el Plan de Movilidad y Espacio Público, entre otros. 
El Plan de Movilidad y Espacio público surgió como respuesta a una política iniciada por la Municipalidad del Cusco. Desde el inicio de su mandato, Carlos Moscoso tenía como prioridad convertir a la ciudad del Cusco en un modelo de ciudad sostenible, en pro de la una verdadera protección del patrimonio. Una de las primeras medidas que se realizó por parte de la municipalidad fue la peatonalización de la plaza de armas del Cusco. Sin embargo, este proyecto se realizó sin una lógica técnica de socialización ciudadana. Como es de esperarse, grupos de empresarios y juntas vecinales salieron a las calles a protestar por el cierre de las vías vehiculares en la plaza. El proyecto causó congestión en las vías alternas. La posición de la plaza y el cierre de sus pistas limitaron la interconectividad entre barrios históricos de la ciudad y se generó molestias entre los vecinos. Con la mayoría de la población en contra de la peatonalización, el proyecto tuvo que ser cancelado.

Por otro lado, el Gobierno Regional estaba realizando un proyecto con el Banco Mundial para la construcción de la Vía Expresa dentro de la ciudad. La municipalidad del Cusco aprovechó que dentro de dicho proyecto existía un excedente de presupuesto y realizó las coordinaciones correspondientes para poder financiar un plan de movilidad financiado con ese dinero. Es así que la municipalidad consiguió el financiamiento para la formulación de un plan articulado sobre movilidad y espacio público. Pero al no contar con la capacidad de endeudamiento requerida por el Banco Mundial, debió hacerse el préstamo a través del Gobierno Regional, lo cual generaría problemas administrativos y de coordinación más adelante. En este caso, hubo delegación de competencias por parte del gobierno regional hacia la municipalidad. Se delegaron competencias técnicas, mientras que el brazo ejecutor de recursos fue Copesco, específicamente a través del Programa de Desarrollo Regional (Proder).

A partir de las entrevistas con especialistas y trabajadores de la municipalidad, realizadas en junio del año 2017, se identificó que el plan tuvo dificultades por la modalidad administrativa de triangulación entre el Banco Mundial, Proder y la Municipalidad del Cusco. Las dos instituciones locales tuvieron problemas para coordinar los pagos de los técnicos que trabajaban dentro del programa, pues la municipalidad y el gobierno regional tienen distintas formas de contratación. Dicho problema generó que los procesos se vuelvan engorrosos y que la entrega de los estudios se demore, lo 
cual, a su vez, generó el desgaste de la imagen del plan.

Más allá de los problemas de administrativos y de coordinación entre instituciones, a través de las entrevistas se ha podido constatar también que la municipalidad no tuvo las capacidades necesarias para implementar un plan de tal envergadura. Los trabajadores de la municipalidad reconocieron que no existían burócratas especializados que pudieran responder a las exigencias técnicas de los gestores del Banco Mundial que trabajaban en la formulación del plan.

Otro problema reconocido por los entrevistados fue que las diferentes oficinas dentro de la municipalidad relacionadas al tema (Oficina de Ordenamiento Urbano, Oficina de Tránsito y Movilidad, Oficina de Gestión del Centro Histórico) no contaban con la información base para la formulación de dicho plan. Este problema técnico dificultó el trabajo de los consultores internacionales, quienes tuvieron que generar su propia información.

A nivel político, si bien es cierto que el alcalde del Cusco mostró su compromiso con el tema, el gobierno regional no tuvo como principal objetivo la formulación de dicho plan. Esta diferencia respecto a la priorización de proyectos generó que el proceso de formulación carezca de legitimación por parte del gobierno regional, lo cual desgastó en mayor medida la imagen de dicho plan frente a la ciudadanía.

Finalmente, otro de los aspectos que se pudo visibilizar en las entrevistas fue el problema generado por la nacionalidad del coordinador general del Plan. José Luis Cañavate es el gestor español encargado del plan. Resulta curioso oír todos los discursos que se han generado alrededor de la nacionalidad del gestor, como por ejemplo «quieren colonizarnos de nuevo», «qué puede saber un español de mi ciudad» 0 «él no puede saber más que los cusqueños». 


\section{Análisis del caso}

Identificar las perspectivas sobre el problema público de los diferentes actores interesados en el diseño del Plan Cusco al 2025 - Movilidad y Espacio Público fue el primer objetivo dentro de esta investigación. En ese sentido, a través del trabajo de campo, se realizaron entrevistas para entender en profundidad dichas perspectivas.

Una de las organizaciones más reacias a la implementación del plan fue la Cámara Regional de Turismo de Cusco (Cartur). Al entrevistar a Carlos Milla, presidente de dicha organización, se pudo comprobar que durante todo el proceso de formulación no se desarrolló un proceso de negociación con Cartur. El empresario afirma que, meses después del cierre de la Plaza de Armas, hubo una serie de reuniones donde fueron invitadas varias organizaciones civiles y público interesado en el tema. Dichas reuniones, según él, no fueron organizadas con el objetivo de asegurar un espacio de retroalimentación y apertura al diálogo entre los diferentes actores. Por el contrario, el coordinador presentó el Plan sin dejar espacio para preguntas u observaciones. Asimismo, durante la entrevista mencionó que cuando los asistentes empezaron a comentar preocupaciones y sugerencias, el personal encargado de la organización de estas reuniones dejó de convocarlos.

Carlos Milla menciona que era claro que los funcionarios de la municipalidad organizaron dichas reuniones para pedir el apoyo de los asistentes, mas no se los trató con el debido respeto, ya que no se consideraron sus recomendaciones y no se los consideró como entes dinámicos que estaban preparados para exigir explicaciones, además de estar capacitados gracias a sus años de experiencia en el sector turismo para remarcar ciertas irregularidades que afectarían a la mayor actividad económica de la ciudad de Cusco. Asimismo, Carlos Milla afirmó que tiempo después de dichas reuniones donde se entregaron documentos que pedían la reconsideración de ciertas decisiones, los miembros de su organización recibieron ataques por las redes sociales donde se los humillaba con temas personales.

Para la Cámara de Turismo de Cusco, además, la falla del plan fue de origen, pues es verdad que había una sobrecarga de vehículos en las vías. Sin embargo, las decisiones que se tomaron fueron de carácter restrictivo y partieron de un falso entendimiento de la movilidad. Sobre la peatonalización 
de la Plaza de Armas, el entrevistado mencionó que dicha herramienta fue usada para revitalizar los centros urbanos que estaban «venidos a menos», pero que en el centro de la ciudad del Cusco sucede exactamente lo contrario: el centro es un espacio con mucha vitalidad, ya que se da una mezcla de actividades que tienen que ver con la vivienda, actividades económicas relacionadas al turismo, así como actividades culturales y administrativas. Para Cartur, peatonalizar para revitalizar es una falacia y los resultados que se han tenido después de este proceso han sido contraproducentes con el bien mayor. Asimismo, la organización ha criticado que se haya restringido el estacionamiento de cualquier vehículo sin antes presentar alternativas, lo cual ha generado problemas con los vecinos y turistas, expulsando al poblador local del centro. Para Cartur, el centro debería ser un lugar de encuentro, pero ahora que se lo ha aislado de la ciudad se ha convertido en una suerte de gueto, donde «gente de mal vivir» se está apropiando de las calles.

Tal como se mencionó en la parte metodológica, para esta investigación se realizó una revisión de los principales diarios de la ciudad del Cusco. Dicha revisión arrojó las principales perspectivas y posiciones de los diferentes actores del problema público.

Para la Municipalidad como institución, el asunto prioritario fue el tema del patrimonio. De acuerdo a lo encontrado en la revisión de prensa, diferentes voceros de la municipalidad sostuvieron que se necesitaba consolidar la peatonización total de la plaza de armas como repuesta a que los carros, en especial los buses de turismo, deterioraban el patrimonio. Para Ismael Sutta, gerente de tránsito y viabilidad de la municipalidad, los objetivos de cualquier acción debían estar dirigidas a «proteger nuestra monumentalidad y recuperar los espacios públicos para el disfrute, además, se debe promover la organización de la ciudad histórica, con el adecuado funcionamiento de la movilidad, de la vida social, cultural y económica, basándose en un agestión eficaz del espacio urbano y en un a distribución coherente entre las diversas funciones y el sistema de transporte» (El Diario del Cusco, 19 de noviembre de 2016).

Para los vecinos del Centro Histórico, el principal reto de las políticas urbanas es crear condiciones adecuadas para revalorar, conservar y preservar el patrimonio cultural, perspectiva que comparten con los funcionarios de la municipalidad. 
Uno de los actores más reacios al plan es la Cámara Regional de Turismo del Cusco. Para esta organización el plan resulta ser un «sueño ajeno e irrealizable en el que se confunden medios (peatonización) con fines (bienestar). Se pretende ilusamente afirmar que el caos actual mejorará limitando aún más las vías disponibles como un enfermo de arterioesclerosis al que se le anulan venas en lugar de adelgazarle la sangre» (extracto de una entrevista para El Diario del Cusco, 19 de noviembre de 2016).

Según la Cámara de Turismo, las acciones que el municipio debió tomar serían cobrar por estacionamiento temporal en las calles, estimular la construcción de playas de estacionamiento, regular integralmente el transporte público y establecer paraderos de buses para el servicio turístico, todo ello en lugar de peatonizar la plaza. Para Cartur, el principal problema de Cusco es la movilidad y con acciones como las que tomó la municipalidad solo se consigue mayor congestión. A la larga, esto lleva a que los cusqueños tengan cada vez más dificultades en el desplazamiento. Ello deteriora la calidad de vida, la cual era precisamente el argumento que sustentaba la visión del plan. Para Cartur, todos los instrumentos que propone el plan respecto a la zona central son los que se usan para atraer turistas y revitalizar centros históricos decaídos y marginados, situación exactamente inversa a la cusqueña.

Otro de los problemas públicos para dicha institución es la construcción clandestina en las laderas de la ciudad, donde se ocupan cuencas, se desfigura el paisaje urbano y se propician aglomeraciones inconexas con calles irregulares y caóticas en las laderas. Esta forma de intervención urbana clandestina no solo ocurre en las periferias de la ciudad, en el centro los propietarios también construyen sin licencia, exceden alturas y coeficientes, desfigurando la monumentalidad de la ciudad.

Según el Instituto de Investigaciones Etnoarqueológicas de Cusco, no hay condiciones favorables para la peatonización en el centro histórico porque no existen vías alternas para el tránsito. La topografía es distinta a la de grandes ciudades extranjeras, lo cual hace que sea imposible llevar a cabo un proceso de peatonización. Para dicha institución, el problema se encuentra en la centralización de actividades e instituciones que se encuentran en el centro de la ciudad. Por lo tanto, lo adecuado sería desconcentrar las actividades y gestiones que se realizan en las instituciones públicas y privadas en el centro. 
Después de conocer las diferentes perspectivas sobre el problema público, podemos señalar que claramente para la mayoría de actores el tema de la protección del patrimonio es fundamental para una ciudad como el Cusco; sin embargo, existe un sinfín de posibilidades para abordar este problema, posibilidades que no han sido escuchadas por las instituciones encargadas del plan.

Analizar las capacidades técnicas y políticas del gobierno local y provincial en la formulación del Plan fue el segundo objetivo que se planteó dentro de esta investigación.

Las capacidades políticas de la municipalidad, entendidas como su habilidad para la representación política y la rendición de cuentas, según la entrevista realizada al regidor Boris Mujica, fracasaron durante el proceso de formulación del plan. El funcionario municipal manifestó que el alcalde había perdido legitimidad frente a la población porque tenía muchos detractores dentro y fuera de la municipalidad y que por esa razón le sería imposible llevar a cabo los objetivos del plan. Según el concejal municipal esto es consecuencia de no convocar a tiempo, así como de no realizar el debido proceso de socialización con las instituciones y actores claves como la Cámara de Comercio del Cusco, organizaciones vecinales, la prensa y los diferentes funcionarios de la municipalidad.

A pesar de esta situación, Miriam Carmona, encargada del área de comunicación dentro del Plan, manifestó que el alcalde, así como todos los funcionarios que trabajaban en la formulación de dicho proyecto, sintieron el respaldo político de instancias del Poder Ejecutivo, tales como el Ministerio de Transporte y el Ministerio de Vivienda, Construcción y Saneamiento. Este respaldo fue materializado en reuniones realizadas con estas entidades, donde además se consideró al Plan de Movilidad y Espacios Públicos del Cusco como el pionero en su tipo dentro del Perú. Asimismo, dichas instancias manifestaron que estaban interesadas en utilizar esta propuesta como un ejemplo para ser replicada en otras ciudades intermedias del Perú.

Sin embargo, a pesar del apoyo de instancias nacionales que ha recibido el alcalde, no se puede olvidar la incapacidad política que ha tenido para negociar con los funcionarios más cercanos a su posición. Los regidores de la 
municipalidad manifiestan que el plan no se llegaría a implementar, pues debe ser llevado a votación y, debido a la poca aceptación que tiene dentro de la escena local, no se tendrían los votos suficientes dentro del consejo municipalidad para llevarlo a cabo.

Respecto a la capacidad técnica dentro del plan, este trabajo se enfocará en la capacidad de coordinación entre las diferentes instancias encargadas de llevarlo a cabo. Además, se hará énfasis en evaluar el debido proceso de formulación del plan, entendido como el correcto uso de los recursos de la municipalidad para el diseño de una política eficaz y eficiente.

En relación a la capacidad técnica del plan, durante el trabajo de campo se entrevistó a Álvaro Cassallo, arquitecto que forma parte del cuerpo técnico del plan. Él manifestó que uno de los problemas más grandes que se tuvo dentro de la formulación del plan fue la difícil coordinación entre la municipalidad y el gobierno regional. Como ya se comentó anteriormente, la municipalidad del Cusco no tiene capacidad de endeudamiento frente al Banco Mundial. Esta es la razón por la cual se tuvo que institucionalizar la función de Proder como instancia encargada de la administración de los fondos económicos del plan. Cabe resaltar que Proder pertenece al Gobierno Regional, lo cual explica que durante el proceso de formulación del plan no haya tenido la mejor disposición para respaldar el trabajo de los funcionarios, ya que como menciona Cassallo, para contratar a un técnico para un trabajo dentro del plan se tenía que realizar un término de referencia que pasaba por la región, luego por Proder y finalmente por el Banco Mundial. Esto dificultó el proceso y retrasó las entregas de los avances al coordinador del Plan. Asimismo, él señala que si se quería cambiar de profesionales por alguna razón era preciso «pensarlo dos veces», ya que dado todo el proceso mencionado anteriormente, era posible quedarse sin personal por unos meses hasta que se aprobaran los términos de referencia de las contrataciones.

Asimismo, dicho funcionario afirma que hay problemas con los pagos y que estos nunca estuvieron al día. Esta situación llevó a que mucho de los funcionarios encargados del plan tuvieran que conseguir otros trabajos aparte de la consultoría para la municipalidad y que el coordinador del plan era consciente de que no se les podía exigir de manera adecuada si es que los pagos no estaban en orden. Cassallo menciona que «ha sido voluntad del equipo poder terminar el plan» dadas todas las situaciones antes expuestas. 
Respecto a la información que la municipalidad debió ser capaz de proporcionar para el correcto proceso de diseño de la política, se entrevistó a José Luis Cañavate, coordinador internacional elegido por el Banco Mundial para realizar el plan. En relación a este tema, el coordinador mencionó que «cada vez que se lograba abrir una puerta, en vez de encontrar los recursos informáticos que se requerían, se encontraban con más preguntas que respuestas». Es decir, la municipalidad y sus diferentes gerencias no eran capaces de brindar información sobre movilidad, vivienda y tránsito del centro de la ciudad. Cañavate afirmó que el nivel de especialización de los funcionarios era básico, muchas veces no entendían lo que se les pedía, y, si existía la data, esta estaba desactualizada. Frente a esta esta situación, el coordinador del plan y su equipo tuvieron que realizar un trabajo para el cual no fueron contratados, la generación de la data.

El coordinador, así como Álvaro Cassallo, principal colaborador, afirmaron que se pidió la contratación de un consultor externo para que se realice el levantamiento de información para así generar data para la formulación del Plan. Pero, como ya se comentó anteriormente, debido a la ineficaz coordinación con Proder, la contratación de estos consultores nunca se llegó a realizar. Fue en ese momento donde el equipo del plan se organizó para cumplir con esta tarea, pues no se podía trabajar sin la información que se les había pedido a las diferentes gerencias dentro de la municipalidad.

Dentro del proceso de formulación del plan, según las entrevistas realizadas, el mayor fallo del equipo de trabajo fue su incapacidad de haber realizado un proceso de socialización a tiempo. En ese sentido, Miriam Carmona, encargada de la parte comunicacional del Plan, mencionó que inicialmente se tuvo una respuesta negativa de la población, pues no se construyeron espacios de debate con esta, además de que no se brindó la información necesaria para evitar malos entendidos. La funcionaria afirma que se hizo las reuniones con las diferentes instituciones interesadas después de que el tema había sido polarizado y la comuna cusqueña había sido mal informada por la prensa local. Respecto a este mismo tema, el regidor Boris Mujica afirma que el plan no cumplió con los parámetros que establece la ley de bases de descentralización, la cual determina que los instrumentos de planificación tienen que pasar por procesos participativos. 
Finalmente, el regidor y los representantes de la Cámara de Comercio y de Turismo del Cusco afirmaron que muchas de las cifras e información que se presentó dentro del plan fueron datos alejados de la realidad. En la elaboración del trabajo de campo, los entrevistados señalaron que dentro de los datos se presentó información absurda como que existía un auto privado por cada dos familias y dos autos por una familia de 5 personas. Ejemplos como este, nos comentan los entrevistados, son los que levantaron sospechas sobre la calidad del trabajo de los funcionarios dentro del diseño del Plan.

\section{Conclusiones}

El Plan Cusco al 2015 - Movilidad y Espacio Público apuntaba a convertirse en un programa único en la región sobre gestión de las ciudades. A pesar de contar con apoyo económico, político y tener el aval de instituciones prestigiosas como el Banco Mundial, al no haber recogido las diferentes perspectivas de los actores implicados durante el proceso de formulación de la política, terminó deslegitimado. A partir de este caso, nos toca reflexionar sobre lo importante que son los procesos de socialización y adaptación al momento de trabajar en políticas públicas.

Al iniciar la investigación, se tenía ciertas hipótesis que finalmente fueron confirmadas en el proceso de trabajo de campo. Temas como la capacidad estatal estuvieron en el centro de la hipótesis. Es bastante común que se espere que dentro de instancias estatales los problemas estén relacionados a problemas técnicos como que no existan burócratas especializados o que la política no tenga suficiente apoyo político. Sin embargo, una de las conclusiones más interesantes que se puede sacar de este trabajo de campo es que nos invita a ver el lado más humano de las políticas que construimos. Es necesario recordar que los beneficiarios de la política, en este caso la comuna cusqueña, no son agentes neutros o pasivos; son personas con un bagaje cultural, historia, aspiraciones, intereses y recursos que en muchos casos los gestores no toman en cuenta al momento de formular planes de esta envergadura. 
Dentro del proceso de formulación del Plan Cusco al 2025, podemos encontrar aspectos positivos como el profundo interés internacional expresado en recursos técnicos y materiales para que se lleve a cabo el plan, el apoyo de parte del gobierno local y nacional, funcionarios internacionales especializados y la legitimidad de ser tratarse de un tema importante para toda la comuna cusqueña. Pero estas variables positivas se vieron deslegitimadas en el proceso de formulación del plan, ya que desde el ámbito técnico no se consideró la totalidad de perspectivas del problema público.

¿Por qué no se consultó a los cusqueños sobre sus expectativas para con su ciudad? Este estudio de caso nos invita a la reflexionar sobre la intrínseca relación que existe entre el espacio urbano, los ciudadanos y sus visiones de lo que significa calidad de vida. En ese sentido, las políticas urbanas no pueden desarrollarse a espaldas de estos detalles culturales. Sabemos que la globalización hace que se democraticen perspectivas sobre ciudades sostenibles y formas de vivir, pero estas ideas hegemónicas no pueden ser desarrolladas sin procesos previos de adaptación a las idiosincrasias locales. Es claro que en una sociedad como la cusqueña, los temas urbanos están intrínsecamente relacionados con temas de patrimonio, lo cual genera especial interés en sus pobladores, ya sea por factores económicos o de identidad.

Dicha reacción nos lleva a replantear el valor que tienen las ciudades y los espacios dentro de nuestra cotidianidad. Las ciudades no son solo lugares donde estamos; las ciudades son la arena donde lo político ocurre, donde los grandes cambios se gestan y por eso mismo cómo perfilamos a nuestras ciudades es un reflejo de cómo somos como sociedad. Al tener ciudades poco inclusivas, violentas o con escasos espacios públicos, revelamos aspectos de cómo somos como sociedad. Este estudio de caso invita a los gestores de políticas urbanas a pensar las ciudades como espacios de convivencia, dotados de variables culturales y de identidad, sin dejar de relacionarlos con las personas que hacen que estos espacios estén vivos. 


\section{Bibliografía}

Bazants, J. (2009) Hacia un desarrollo urbano sustentable: Problemas y criterios de solución. MéxicoD.F.: Limusa.

Blanco, J. (2015). Hacia el diseño y gestión de barrios sustentables en Chile. Revista INVI, 31(86), 203-214. https://doi.org/10.4067/s0718$\underline{83582016000100008}$

Carrión, F. (2007) «El desafío político de gobernar la ciudad» Nueva Sociedad, 212, 36-52

Casanueva, P. y Fernández, M. (eds.) (2010) Estrategias medioambientales y sostenibilidad urbana. Edición que reúne los trabajos presentados en el I Ciclo de Conferencias sobre Desarrollo Sostenible. Universidad Europea Miguel de Cervantes.

Chion, M. (2002) «Dimensión metropolitana de la globalización: Lima a fines del siglo XX» Revista EURE, 28(85), 71-87. https://doi.org/10.4067/s0250-71612002008500005

Córdova, A. (2013) El transporte urbano como factor determinante en la sostenibilidad ambiental de las ciudades: Caso de Lima. En Borsdorf, A. (ed.). Forschen in Gebirge. Investigating the Mountains. Institut für Interdisziplinäre Gebirgsforschung. pp. 211-232.

Dextre, J. (2001) Curso de ingeniería de tráfico, políticas de transporte y desarrollo sustentable. Lima: Colegio de Ingenieros del Perú.

El Diario del Cusco (2016) Cámara de Turismo de Cusco -CARTUC expresa su desacuerdo con el denominado "Plan Cusco 2025. 19 de noviembre.

Girardet, H. (2001) Creando ciudades sostenibles: Creating sustainables cities. Valencia: Ediciones Tilde. 
Instituto Social de Vivienda y Hábitat Alcaldía de Medellín (ed.) (2014)

Carta Medellín: Sobre el porvenir humano de las urbes del mundo. Medellín. Séptimo Foro Urbano ONU-Habitat.

Martínez, M. (2012) «La pérdida de urbanidad: Lima en el boom de los centros comerciales del siglo XXI» Revista de la Facultad de Arquitectura y Urbanismo, 7(7), 58-69

Mattos, C. (2002) «Transformación de las ciudades latinoamericanas:

¿Impactos de la globalización?» Revista EURE, 28(85), 5-10

https://doi.org/10.4067/s0250-71612002008500001

Melgosa, J. (2001) Turismo y sostenibilidad en las Ciudades Patrimonio de la Humanidad: principios, marco e instrumentos de actuación. Salamanca: Ministerio de Educación, Cultura y Deporte.

Parnreiter, C. (2002) «Ciudad de México: el camino hacia una ciudad global» Revista EURE. 27 (85), 89-119 https://doi.org/10.4067/s0250$\underline{71612002008500006}$

Stadel, C. (2014) Ciudades medianas y aspectos de la sustentabilidad urbana en la región andina. Espacio y Desarrollo. Pontificia Universidad Católica del Perú. Lima, 12, pp. 25-43.

Subirats, J y Dente, B. (2014) Decisiones públicas: Análisis y estudio de los procesos de decisión en políticas públicas. Barcelona: Ariel.

Troitiño, L. (2011) Ciudades Patrimonio de la Humanidad de España: dinámica turística en tiempos de crisis (2000 - 2009). Madrid: AGE.

Velz, P. (2000) Le nouveau monde industriel. Paris: Gallimard. 


\section{ANEXO: Guía de entrevistas}

\section{Funcionarios y regidores}

Perfil del entrevistado: Funcionarios de la municipalidad, equipo de trabajo del Plan y regidores de la municipalidad.

Objetivo 1: Analizar las características del proceso de formulación del Plan Cusco al 2025-Movilidad y Espacio Público

1. ¿Cómo surgió la iniciativa del proyecto?

2. ¿Cuáles fueron los canales de comunicación durante el proceso de formulación del plan?

3. ¿Qué instituciones estuvieron presentes en el proceso de formulación del plan?

4. Coménteme el proceso de negociación durante la formulación del plan

5. ¿Cuál es su posición frente a las líneas de acción del plan?

Objetivo 2: identificar las perspectivas sobre el problema público de los diferentes actores.

1. ¿Cuál es la perspectiva del problema público de la institución en la que trabaja?

2. ¿Quiénes son los otros actores influyentes dentro de la formulación del Plan?

3. ¿Conoce la perspectiva del problema público que manejan estos actores?

4. ¿Qué opina sobre estas perspectivas?

5. ¿Qué perspectiva del problema público cree que se debería priorizar? 
Objetivo 3: analizar las capacidades técnicas y políticas de las diferentes instituciones implicadas en la formulación e implementación del plan cusco al 2025 - movilización y espacio público.

1. ¿Cuáles fueron los despachos específicos que se encargaron de trabajar el plan?

2. ¿Cuáles fueron sus funciones dentro de la formulación?

3. ¿Cuál fue la dinámica de priorización de opiniones dentro del plan? ¿Cuál de las instituciones tenía más poder?

4. ¿Cree que su institución tiene la capacidad para desarrollar un plan de esta magnitud? ¿Qué deficiencias puede identificar?

\section{Comuna cusqueña}

Perfil del entrevistado: Vecinos, empresarios y medios de comunicación

Objetivos: Conocer la perspectiva de los actores sobre el problema público, entender las razones por las cuales los entrevistados consideran que el plan no debería realizarse

1. ¿Cuál es su perspectiva sobre el problema generado alrededor del plan?

2. ¿Fue partícipe de algún proceso de negociación?

3. ¿Cuáles son los canales por los cuales la municipalidad le informó del plan?

4. ¿Por qué cree que el plan despierta tantos conflictos?

5. ¿Considera que la municipalidad está capacitada para llevar un plan de este tipo?

6. ¿Qué recomendaciones le daría a la municipalidad? 